\title{
Implementation of the Contensou-Erismann Model of Friction in Frame of the Hertz Contact Problem on Modelica
}

\author{
Ivan Kosenko Evgeniy Aleksandrov \\ Russian State University of Tourism and Service, Department of Engineering Mechanics \\ Glavnaya str. 99, Cherkizovo-1, Moscow reg., 141221, Russia
}

\begin{abstract}
An approximate model to compute resulting wrench of the dry friction tangent forces in frame of the Hertz contact problem is built up. An approach under consideration develops in a natural way the contact model constructed earlier. Generally an analytic computation of the integrals in the Contensou-Erismann model leads to the cumbersome calculation, decades of terms, including rational functions depending in turn on complete elliptic integrals. To implement the elastic bodies contact interaction computer model fast enough one builds up an approximate model in the way initially proposed by Contensou.

To verify the model built results obtained by several authors were applied. First the Tippe-Top dynamic model is used as an example under testing. It turned out the top revolution process is identical to one simulated with use of the set-valued functions approach. In addition, the ball bearing dynamic model was also used to verify different approaches to the tangent forces computational implementation in details. A model objects corresponding to contacts between balls and raceways were replaced by ones of a new class developed here. Then the friction model of the approximate Contensou type embedded into the whole bearing dynamic model was thoroughly tested.
\end{abstract}

Keywords: Hertz contact model; Contensou simplified model; Contensou-Erismann model; Vil'ke model; Tippe-Top; ball bearing model

\section{Introduction}

To make a contact model for the multibody dynamics more accurate and simultaneosly more efficient using the Hertz contact problem as a frame one has to develop an approach taking into account nature of the tangent forces acting along a contact spot area. The simplest case one could encounter in this way is one of the dry friction forces distributed over the elliptic area arising in the Hertz model. It is known as the Contensou-Erismann friction model $[1,2]$.

The model assumes the resulting wrench of the dry friction tangent forces. The wrench consists of the total friction force and the drilling friction torque. An approach under consideration continues in a natural way the contact model development started earlier [3]. The normal contact force distribution is determined by the Hertz model while the tangent forces on an elemental level satisfy the Amontons-Coulomb law for dry friction.

The dry friction force and torque are integrated over the contact elliptic spot thus composing the resulting wrench. Generally an analytic computation of the integrals mentioned leads to the cumbersome calculation including decades of terms depending on rational functions depending in turn on complete elliptic integrals.

To keep an accuracy and to make the model fast enough an approach proposed initially by Contensou [1] is built up. The model under construction is one derived from the Contensou simplified model in the following directions: (a) the model is anisotropic: total friction forces along the contact ellipse axes are different; (b) for the translatory and almost translatory relative motions one uses the Amontons-Coulomb friction law regularization [4]; (c) the approximate model for the drilling torque also is under construction.

\section{Problem Formulation}

The Hertz problem solution [5] to a normal pressure distributed over the contact area of elliptic shape is defined [6] by the formula

$$
\sigma(x, y)=\frac{3 N}{2 \pi a b} \sqrt{1-\frac{x^{2}}{a^{2}}-\frac{y^{2}}{b^{2}}},
$$

where $N$ is the total force of normal pressure, $a$ and $b$ are the contact spot ellipse semi-major and semi-minor 
axes respectively, see Figure 1, Pxyz is the contact local coordinate frame oriented such that the $x$-axis is directed along the ellipse semi-major axis. All three values: $N, a$, and $b$ supposed already computed by the Hertz algorithm [3].

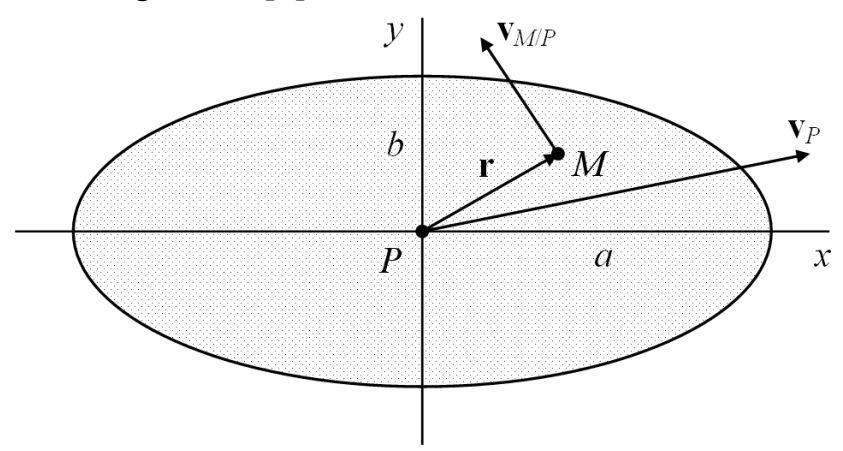

Figure 1: The contact spot area

The body $A$ supposed "below/behind" the picture of Figure 1 plane while the body $B$ supposed "above/in front of" it. In addition, all the forces under computation here supposed to act to the body $B$ from the body $A$. Consider a method to compute a wrench consisting of the tangent force $\mathbf{F}=F_{x} \mathbf{i}+F_{y} \mathbf{j}$ and the resulting torque $\mathbf{T}=T_{z} \mathbf{k}$ arising due to couple of dry friction forces distributed over the contact area. This latter one usually called a drilling friction torque.

According to the dry friction definition the tangent stress $\tau(x, y)$ at the contact spot any point $M(x, y)$ is computed by the formula

$$
\boldsymbol{\tau}(x, y)=-f \sigma(x, y) \frac{\mathbf{v}_{M}(x, y)}{\left|\mathbf{v}_{M}(x, y)\right|},
$$

where $f$ is the dry friction coefficient, $\mathbf{v}_{M}(x, y)$ is the relative slip velocity of the body $B$ with respect to (w. r. t.) body $A$ at the geometric point $M$. The right hand side of Eqn. (1) isn't regular. Because of that the set-valued functions calculus is applied frequently to the problems including dry friction [7]. Let us try to build up a computational procedures for the dry friction problems staying in frame of classical calculus and using the known results $[8,9]$ on asymptotic closeness for an exact and an approximate problems.

Assuming the bodies $A$ and $B$ to be rigid from the kinematic viewpoint the body $B$ relative motion, along the contact spot plane, is an instant planar (the relative velocity normal component supposed to participate only in the normal force computation), and subsequently field of velocities over the spot is defined by the known Euler formula

$$
\mathbf{v}_{M}(x, y)=\mathbf{v}_{P}+\mathbf{v}_{M / P}(x, y)=\left(\begin{array}{c}
v_{P x}-\omega_{z} y \\
v_{P y}+\omega_{z} x \\
0
\end{array}\right),
$$

where $\mathbf{v}_{M / P}(x, y)=[\boldsymbol{\omega}, \mathbf{r}(x, y)]$, and $\mathbf{r}(x, y)=x \mathbf{i}+y \mathbf{j}$ is the current point $M(x, y)$ radius vector within the contact spot, see Figure 1. The ellipse central point relative slip velocity $\mathbf{v}_{P}$ is represented as follows

$$
\mathbf{v}_{P}=v\left(\begin{array}{c}
\alpha \\
\beta \\
0
\end{array}\right)=v \mathbf{w}, \quad \alpha=\cos \psi, \quad \beta=\sin \psi,
$$

where $v$ is a relative slip velocity value at the point $P$, and $\psi$ is the angle between the axis $P x$ and vector $\mathbf{v}_{P}$. According to the Contensou-Erismann model [1,2] to compute the dry friction total force and torque vectors one has to evaluate integrals over the contact elliptic area in the following way

$$
\mathbf{F}=\iint \boldsymbol{\tau}(x, y) d x d y, \mathbf{T}=\iint[\mathbf{r}(x, y), \boldsymbol{\tau}(x, y)] d x d y .
$$

For the further use it is suitable to introduce the dimensionless velocity $u=v / a \omega_{z}$ of relative slipping at the point $P$ instead of a dimensioned one.

\section{Theoretical Background}

It turned out the friction total force and drilling friction torque components are regular functions of the relative sliding (dimensionless) velocity $u$, relative angular velocity supposed fixed parameter here, at a center of the ellipse such that for the exact force $\mathbf{F}$ and torque $\mathbf{T}$ we have

$$
\mathbf{F}(u)=\mathbf{F}_{0}^{\infty}+\mathbf{O}\left(u^{-2}\right), \quad \mathbf{T}(u)=\mathbf{T}_{1}^{\infty} u^{-1}+\mathbf{O}\left(u^{-2}\right)
$$

as $u \longrightarrow \infty$, and

$$
\mathbf{F}(u)=\mathbf{F}_{1}^{0} u+\mathbf{O}\left(u^{2}\right), \quad \mathbf{T}(u)=\mathbf{T}_{0}^{0}+\mathbf{O}\left(u^{2}\right)
$$

as $u \longrightarrow 0$. Here $\mathbf{F}_{0}^{\infty}, \mathbf{T}_{1}^{\infty}, \mathbf{F}_{1}^{0}, \mathbf{T}_{0}^{0}$ are constant vectors defining the approximate model. The vectors $\mathbf{F}_{1}^{0}, \mathbf{T}_{0}^{0}$ depend on complete elliptic integrals of the first and the second kind depending in turn on the contact ellipse eccentricity in the following way

$$
F_{1 x}^{0}=-\alpha A u, \quad F_{1 y}^{0}=-\beta B u, \quad T_{0 z}^{0}=-C
$$

with the constants

$$
\begin{aligned}
A & =\frac{3}{2} \frac{\mathbf{K}(e)-\mathbf{E}(e)}{e^{2}}, \\
B & =\frac{3}{2}\left(\mathbf{K}(e)+\frac{\mathbf{E}(e)-\mathbf{K}(e)}{e^{2}}\right), \\
C & =\frac{3}{8} \mathbf{E}(e),
\end{aligned}
$$


where $\mathbf{K}(e), \mathbf{E}(e)$ are complete elliptic integrals of the first and second kind respectively. Remark that really the values $A, B, C$ are a variable functions of time because the contact ellipse eccentricity $e$ can vary while the simulation process.

Note here the dry friction total wrench simplified model doesn't require any noticeable computational resources because complete elliptic integrals mentioned are already calculated while computing the total normal force according to the Hertz model.

As Contensou [1] remarked the main effect in the Contensou-Erismann dry friction model if the contact area is non-zero besides the drilling friction torque arises is that the total friction force decreases monotonically to zero as a function of $u$.

On the other hand one can easily note from (5) that a steepness of the total friction force change, as a function of $v$, grows as $a \omega_{z} \longrightarrow 0$. In this case either contact spot area decreases to zero or the drilling angular velocity vanishes. Finally, for the value $a \omega_{z}$ small enough and $A, B \geq a \omega_{z} / \delta$, where $\delta$ is a regularization parameter for the case of dry friction, we have the almost point contact case already implemented earlier [4] as a dry friction model regularization. Thus in the current simplified Contensou model resulting computer model always uses the "regular" case of the friction force decreasing, though sometimes steep, to zero. Taking into account that according to (4) for $u$ large enough the simplified friction force differs from its Coulomb's value by the magnitude of the second order of smallness and following the Contensou proposal [1] let us simplify our model such that the friction force supposed to be of the Amontons-Coulomb type for $u \in\left[u_{x, y}^{*}, \infty\right)$ and linear one for $u \in\left[0, u_{x, y}^{*}\right)$. Note, the friction force has an anisotropy here: constants $u_{x}^{*}$ and $u_{y}^{*}$ along axes $P x$ and $P y$ respectively are in general different. Evidently, we can find these values from equations

$$
A u_{x}^{*}=1, \quad B u_{y}^{*}=1 .
$$

Now we can represent the Contensou approximate model for the (dimensionless) functions $F_{x}(u), F_{y}(u)$, $T_{z}(u)$ as follows

$$
\begin{aligned}
& X_{C}(u)=-\alpha\left\{\begin{array}{lll}
A u & \text { for } & u \in\left[0, u_{x}^{*}\right), \\
1 & \text { for } u \in\left[u_{x}^{*}, \infty\right),
\end{array}\right. \\
& Y_{C}(u)=-\beta\left\{\begin{array}{lll}
B u & \text { for } u \in\left[0, u_{y}^{*}\right), \\
1 & \text { for } u \in\left[u_{y}^{*}, \infty\right),
\end{array}\right. \\
& T_{C}(u)=\left\{\begin{array}{lll}
-C & \text { for } u \in\left[0, u_{z}^{*}\right), \\
T_{1 z}^{\infty} u^{-1} & \text { for } u \in\left[u_{z}^{*}, \infty\right),
\end{array}\right.
\end{aligned}
$$

where $u_{z}^{*}$ is a sewing point for the horizontal "shelf" of height $C$ and a branch of the hyperbola decreasing at

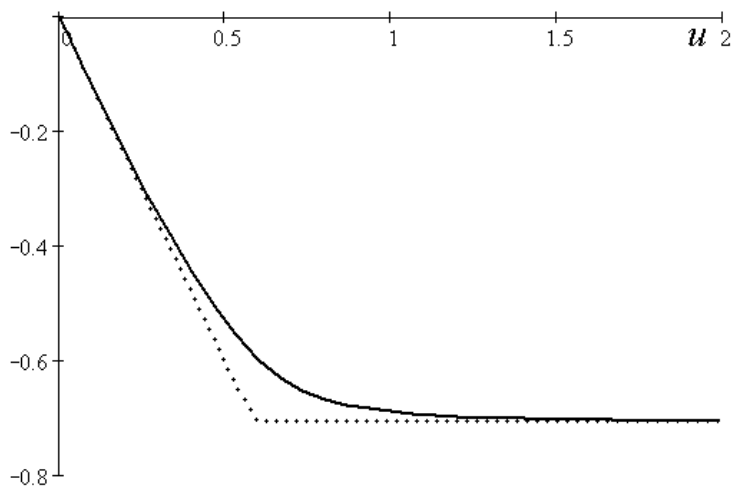

Figure 2: $x$-components of the force vector for exact and approximate models (similar picture for $y$ components)

infinity and being defined by the function $T_{z}^{\infty}(u)$. An equation specifying the value $u_{z}^{*}$ has the form

$$
C=-\frac{T_{1 z}^{\infty}}{u_{z}^{*}}
$$

Comparison of graphs for the Contensou-Erismann model functions is represented in Figures 2 and 3. Functions of the exact model correspond to solid lines, and ones of the approximate model correspond to the dotted lines.

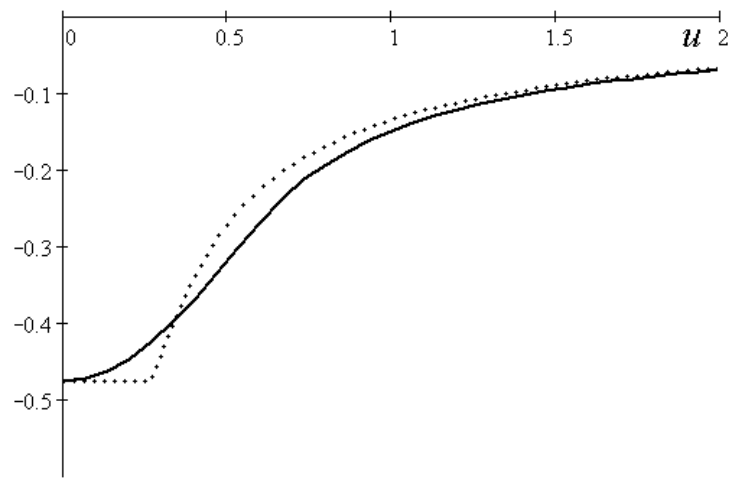

Figure 3: The drilling friction torque for exact and approximate models

If while the simulation process all the values $u_{x}^{*}, u_{y}^{*}$, $u_{z}^{*}$ found become less than $\delta / a \omega_{z}$ then we arrive at the regularized Coulomb model implemented earlier. Thus the approximate Contensou model implemented here really is a simplest generalization of the regularized Coulomb one mentioned regularizing it even more by introducing the parameters $u_{x}^{*}, u_{y}^{*}, u_{z}^{*}$ enhancing initial use of the parameter $\delta$. Such an improvement simply is a consequence of the contact spot existence in the exact model. Thus as a result, with the 
exception of the cases of $a=0$ and $\omega_{z}=0$, we can avoid use the set-valued functions being able to apply the procedures of classical calculus.

The approximate model under construction here has several differences from a piece-wise linear approximation built up in the paper [1]:

(a) the model is anisotropic and is suitable for the elliptic contact area of any eccentricity;

(b) for the cases of instant translatory and almost instant translatory bodies in the contact relative motion with the conditions

$$
u_{x}^{*} a \omega_{z}<\delta, \quad u_{y}^{*} a \omega_{z}<\delta, \quad u_{z}^{*} a \omega_{z}<\delta
$$

fulfilled simultaneously we apply the dry friction regularization proposed in [8] and [11];

(c) the approximate model used also for the drilling friction torque.

Computations show the force/torque expressions represented here give an approximation of the Contensou-Erismann model more accurate in compare with the linear-fractional approximation satisfying boundary conditions at zero and infinity. If we use the Pade approximations with the polynomials of the second and third degrees [12] then the resulting accuracy is improved but computations become more significant.

It is known [13] the V. G. Vil'ke formula gives an approximation for the contact interaction normal elastic force decent enough in a wide range of eccentricities. Computer implementation of such a model runs noticeably faster than the implementation of the exact Hertz model. The main reason for that is a necessity in the latter case to resolve the transcendental equation

$$
\frac{1}{2} \frac{\mathbf{K}(c)}{\mathbf{K}^{\prime}(c)}-(1-c)=g \quad(0 \leq c<1,0<g \leq 1)
$$

w. r. t. $c=e^{2}$ which is the contact spot eccentricity squared. Here we use the elliptic integral modulus squared $c$ as an argument of complete elliptic integral of the first kind, as it has been done in [14].

The V. G. Vil'ke algorithm to compute the normal contact force doesn't require to know the current value of $c$ but the Contensou-Erismann anisotropic friction model does. To keep the gain has been gotten while the normal force calculation and don't waste the computer time to resolve the equation (6) this time to compute the tangent friction force it turned out to be possible that the solution mentioned can be reduced to the explicit linear formula once applied.
After the value $c=c_{*}$ needed has been computed then to find the values $A, B, C$ mentioned above we should calculate complete elliptic integrals of the first and second kind using theta-functions [14]. First of all for any $c_{*} \in[0,1)$ one can use the expansion

$$
\theta_{3}(q)=1+2 \sum_{n=1}^{\infty} q^{n^{2}}
$$

with fast conversion such that complete elliptic integral of the first kind can be computed by the formula

$$
\mathbf{K}(c)=\frac{\pi}{2} \theta_{3}^{2}(q),
$$

where nome $q$ is calculated with a very high accuracy using equations $[14,15]$

$$
\begin{gathered}
\varepsilon=\frac{1}{2} \cdot \frac{1-(1-c)^{1 / 4}}{1+(1-c)^{1 / 4}}, \\
q=\varepsilon+2 \varepsilon^{5}+15 \varepsilon^{9}+150 \varepsilon^{13}+1707 \varepsilon^{17}+\ldots,
\end{gathered}
$$

and the terms enumerated above are sufficient for the accuracy level of order not less than one for the value $1 / 2^{21}$.

It is convenient for complete elliptic integral of the second kind to use the formula [16]

$$
\mathbf{E}(c)=\frac{2-c}{3} \mathbf{K}(c)+\frac{\pi^{2}}{\mathbf{K}(c)}\left[\frac{1}{12}-2 \sum_{n=1}^{\infty} \frac{q^{2 n}}{\left(1-q^{2 n}\right)^{2}}\right] .
$$

If the value of $c$ is small then to regularize the expression

$$
\frac{\mathbf{E}(c)-\mathbf{K}(c)}{2 c}=\frac{d \mathbf{E}}{d c}
$$

one can use hypergeometric expansions converging well enough in this case [17].

\section{Implementation Specifics}

According to experience while developing the models for elastic contacting of rigid bodies interactions in the multibody dynamics a flexibility provided by Modelica can be used to utilize a wide variety of different properties concerning a contact of solids. The properties are mainly of the following categories:

(a) geometric properties for surfaces in vicinity of the contact spot (gradients of the functions defining surfaces, their Hesse matrices);

(b) a model to compute the contact area dimensions and normal elastic force; 


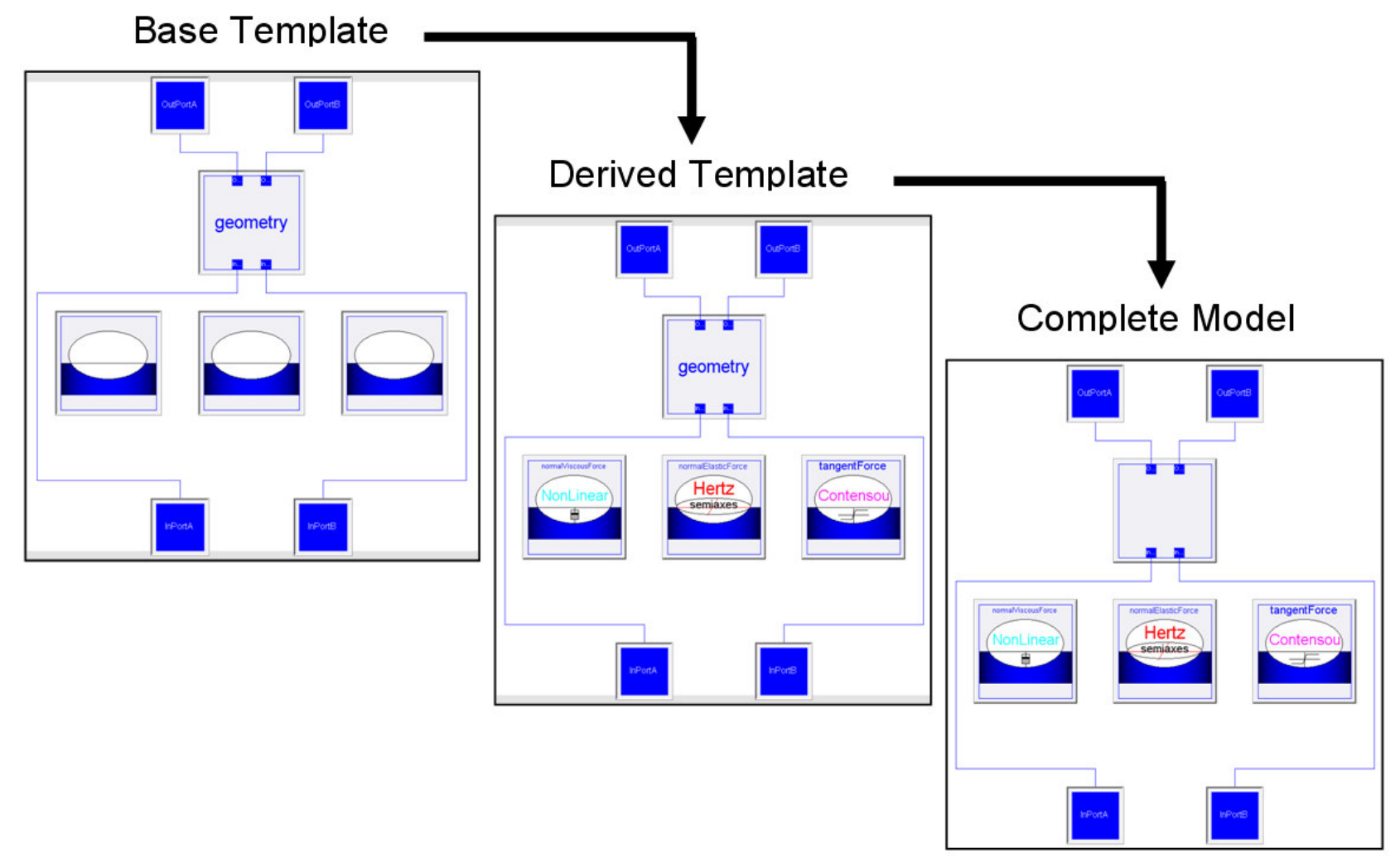

Figure 4: The model of mechanical contact by stages of inheritance.

(c) model for the normal viscous force of resistance;

(d) model for the tangent forces along the plane of the contact area.

A submodel of the geometry properties is to describe analytically algebraic surfaces of the structure complex enough. To implement the normal force computation one can choose from at least two approaches: the Hertz model and its volumetric modification. Force of viscous resistance also can be modeled in several different ways: linear, non-linear, etc. In the models for tangent forces one can adopt either "simplest" approaches based on the Amontons-Coulomb friction or more complex ones represented by the ContensouErismann, and other models.

While developing a mechanical contact model architecture we used the base class Constraint described earlier [18] as a starting point to construct its inheritor ContactConstraint Template being simultaneously a base class of new family of models to simulate mechanical contacts. Really this class is a base template represented as a container having four "sockets" to instantiate there the specific parameter classes of four types enumerated above, see its visual model in Figure 4 at a top left corner, and its Modelica code can be outlined as follows

partial model ContactConstraintTemplate extends Constraint;

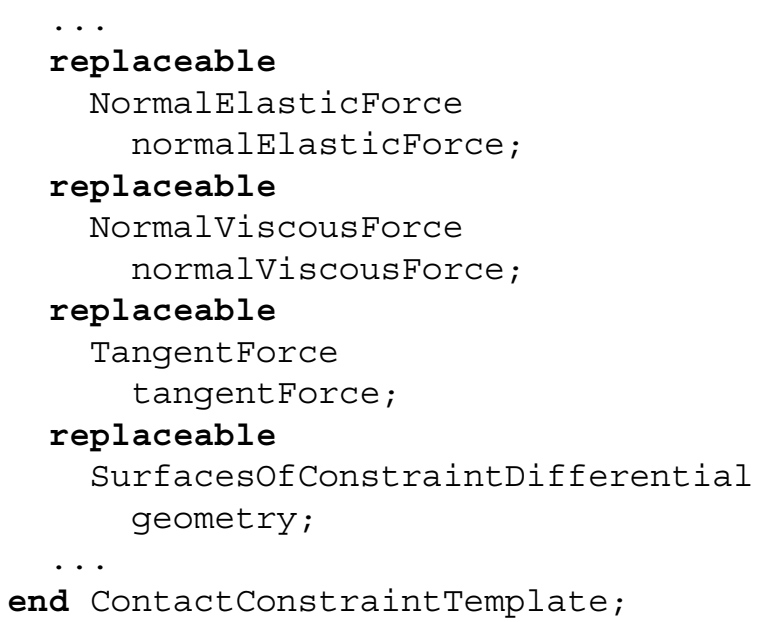

To develop complete model one can move along different ways. Class parametrization implemented in Modelica is the facility in line to apply to the problem under description. In our case we have four class parameters corresponding to the submodel categories enumerated above. An example to construct specific contact interaction model see in Figure 4. The example includes two stages of inheritance:

1. to derive a template with the forces models, namely: the Hertz model for normal force, nonlinear viscous force, the Contensou-Erismann model for the dry friction forces (to "fill in" three sockets in the middle of the base template visual 
model, see the derived template visual model at a central position of the Figure 4);

2. to complete the whole construct one should define a specific geometry submodel for the surfaces in contact (to "seal" the socket for geometry properties, see the complete visual model at a bottom right corner of the Figure 4).

The Modelica code for the intermediate derived template can be represented in the following way

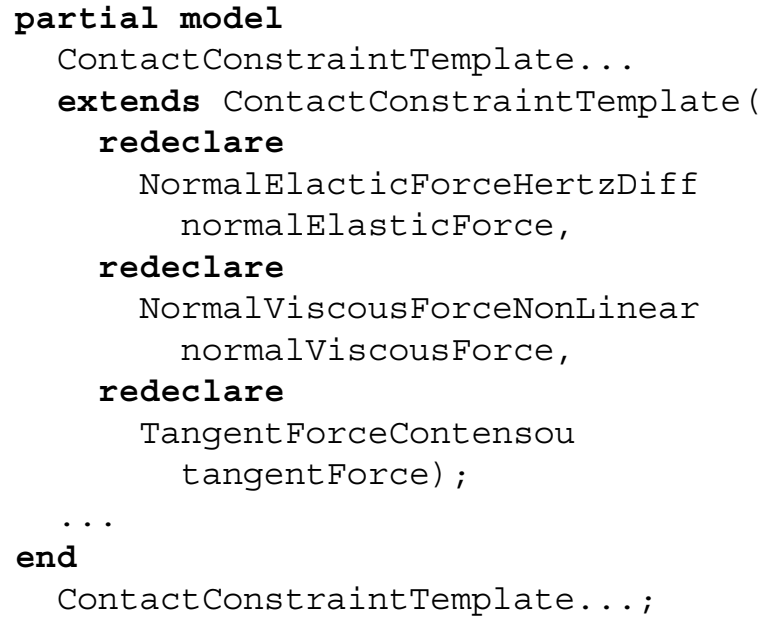

On all the stages of inheritance the templates considered have an internal information interconnections between the submodels to be instantiated. These interconnections are implemented via the set of equations hidden behind the visual models and can vary for different models requiring different variables for the algorithms to compute normal and tangent forces of the complete model. So the whole picture remind us known construct of a card with the sockets and the interconnection wiring in its internal layers as a base template, and a chips to be instantiated in the sockets as a models of four types from above. With one exclusion: we have the derived template playing a role of additional card with its own additional wiring servicing already instantiated models "covering the card" of the base template.

One can remark finally an approach under presentation allows us to create and to change fast enough different types of an elastic contact models while developing the multibody dynamics systems simulators.

\section{Numeric Experiments}

The tangent forces model under presentation here has been verified by two stages: (a) for the case of circular contact; (b) for the case of elliptic non-circular contact. The known Tippe-Top dynamical model was

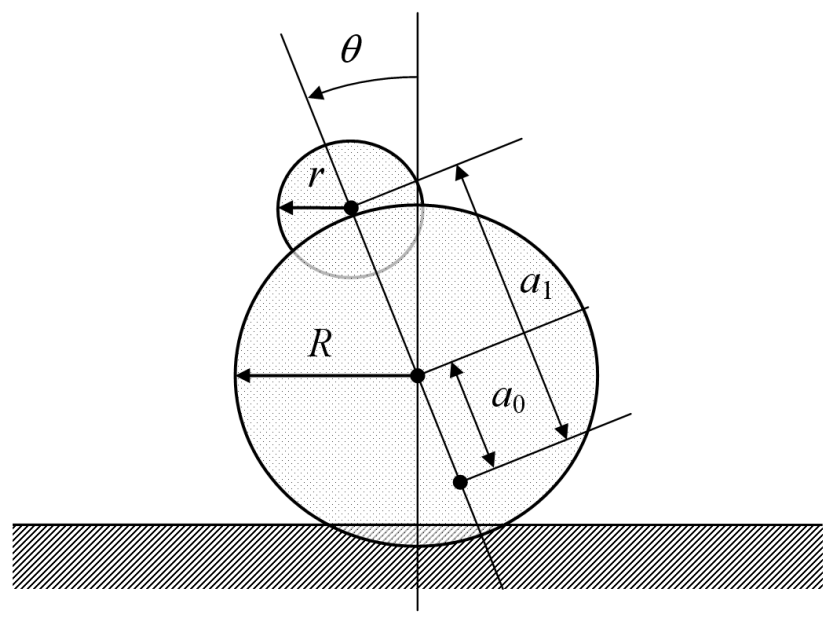

Figure 5: The Tippe-Top geometric properties

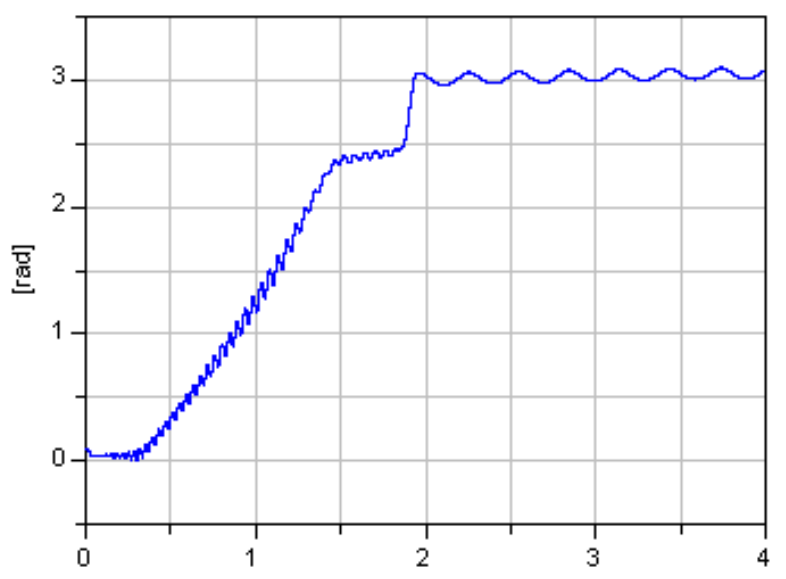

Figure 6: The top axis of symmetry evolution

investigated as an example of the first case. All the parameters and initial conditions are exactly the same as in the paper [19] whose authors got these data in turn from the work [20]. The only difference is that in our case we considered an unrestricted problem with the contact ellipse, including depth of penetration and normal force, being computed dynamically.

The top body, supposed geometrically rigid, composed by two balls, Figure 5, one of larger radius $R=1.5 \cdot 10^{-2} \mathrm{~m}$, and another, smaller, one of the radius $r=0.5 \cdot 10^{-2} \mathrm{~m}$. The top mass center location supposed "under" the larger ball geometric center on its axis of symmetry at a distance of $a_{0}=3 \cdot 10^{-3} \mathrm{~m}$ and at the distance of $a_{1}=16 \cdot 10^{-3} \mathrm{~m}$ to the smaller ball center. The top mass is equal to $m=6 \cdot 10^{-3} \mathrm{~kg}$. The top body supposed dynamically symmetric, and the central principal moments of inertia are the following: an equatorial moment equals to $8 \cdot 10^{-7} \mathrm{~kg} \cdot \mathrm{m}^{2}$, and a polar one has the value of $7 \cdot 10^{-7} \mathrm{~kg} \cdot \mathrm{m}^{2}$. A material the top and the horizontal floor the top rolls on are made 


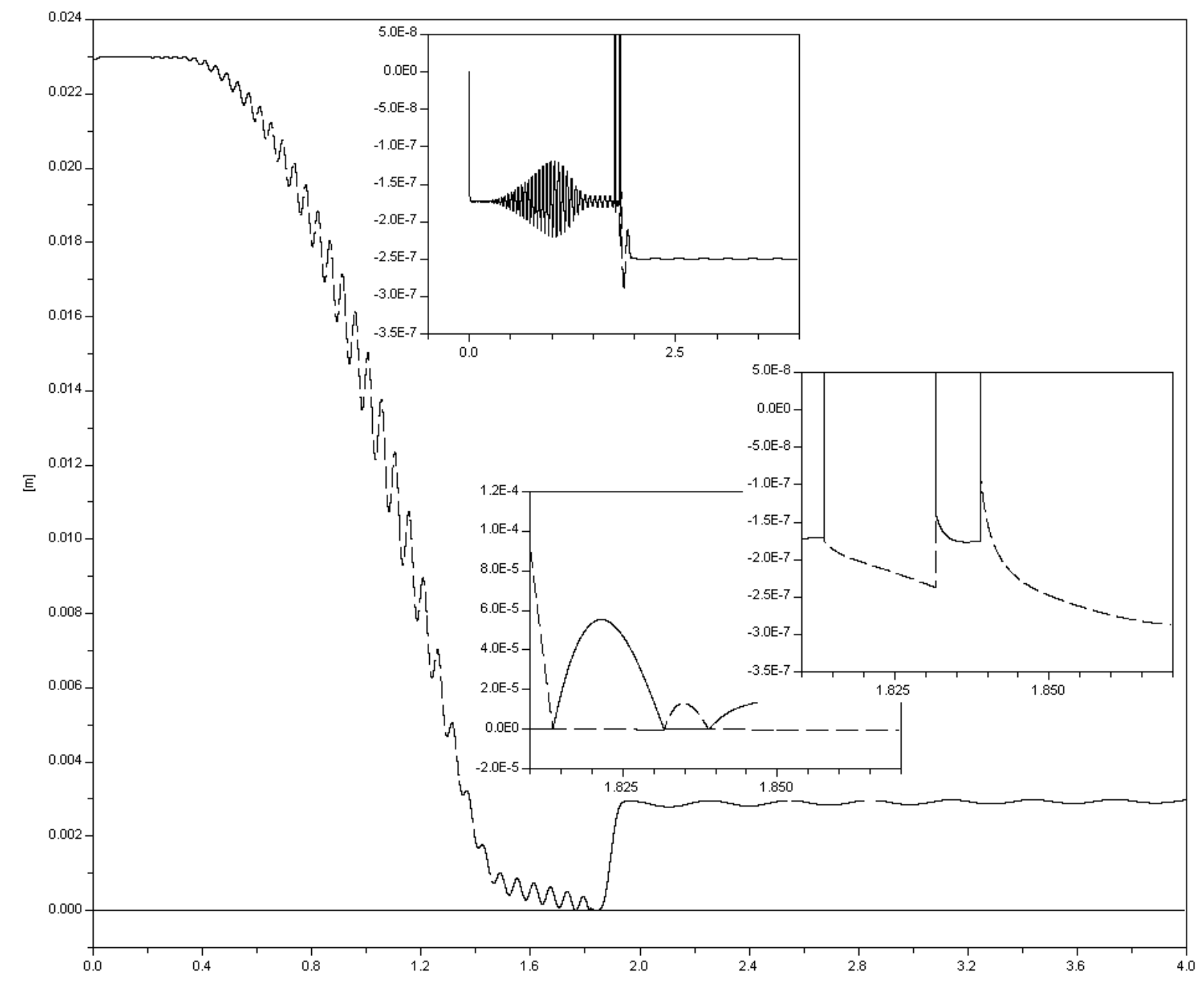

Figure 7: The contact indicators evolution. Some fragments zoomed in and rescaled.

of the wood with Young's modulus $E=9.1 \cdot 10^{9} \mathrm{~N} / \mathrm{m}^{2}$. If we suppose the Poisson ratios as 0.3 then an effective Young's modulus has to be $E_{*}=5 \cdot 10^{9} \mathrm{~N} / \mathrm{m}^{2}$ just as in [19]. The dry friction coefficient supposed to be equal to the value $f=0.3$.

The top center of mass supposed resting at initial instant of motion. Besides the top itself, more accurately its larger ball, assumed without any initial penetration with the horizontal surface. The smaller ball is located on the upper hemisphere of the larger ball, and initially the top axis of symmetry bends w. r. t. vertical by the angle $\theta_{0}=0.1 \mathrm{rad}$. Initial angular velocity $\omega_{0}=180 \mathrm{~s}^{-1}$ is the same as in [19] and directed along axis of the top symmetry.

Note that in [19] contact problem is interpreted as usual in so to speak "restricted" sense: the contact area supposed constant and predefined corresponding to the normal force of the static equilibrium. This assumption concerns the contacts for both the balls with the same contact area radius. Actually, while motion the normal reaction force, being implemented here by elastic forces, changes. Then the contact spot changes also, and so for its radius. Thus the top really undergoes the vertical microvibrations. And, as one can see from the above simplified model, the drilling friction torque also changes causing in general a consequences for the top motion.

In the model under development here we consider an unrestricted contact problem that is the normal force is computed from the Hertz (or V. G. Vil'ke) model with addition of some nonlinear viscous term. Simultaneously the contact area is computed too. Then all the data have been gotten are used to calculate the tangent force and the drilling friction torque in frames of the simplified Contensou model.

Remarkably, a computational experiment showed the top revolution from "feet", the larger ball in contact, to "head", the smaller ball in contact, scenario obtained in [19] using another approach to the problem, based on the set-valued functions calculus, repeated in our model with a high degree of accuracy. One can get an access to the paper [19] visiting, for instance, the 


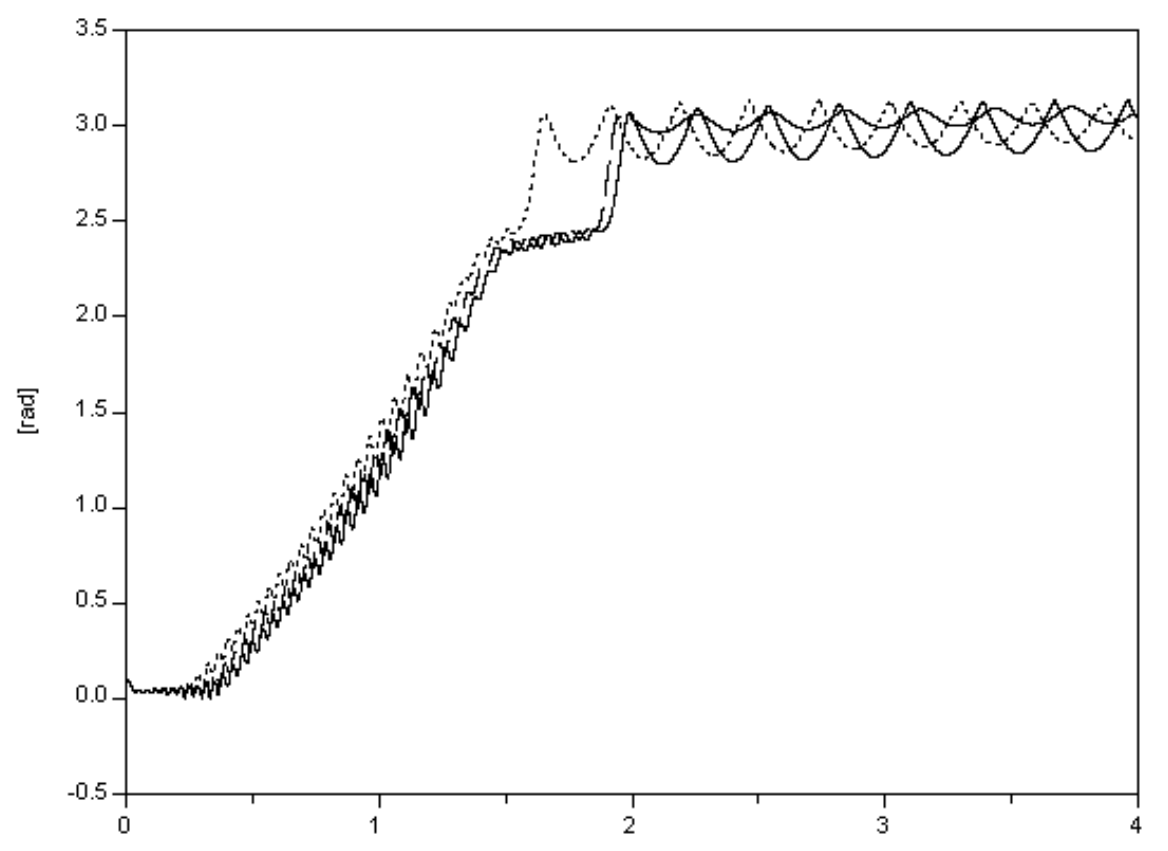

Figure 8: Comparison of three models

one of the authors Home Page, see [21]. Graph in Figure 6 illustrates well the Tippe-Top revolution process: similar to [19] it shows the $\theta(t)$ angle evolution. If we compare this plot with one from [19] then soon the complete identity can be observed. The only difference is that in Figure 6 one can find additional vibrations of small amplitude evidently existing due to elastic compliance in direction normal to the contact area. Similar identity show the curves of the contact indicators for the balls the top composed of and the horizontal surface, see Figure 7. The indicator for the pair (larger ball, floor) marked by the solid line while the (smaller ball, floor) contact indicator pictured as a dashed curve.

Really an indicators are the distances between an opposite points for the surfaces being tracked for contact. The indicator is strictly positive if contact is absent. Otherwise it is less than (if the bodies are in a state of mutual penetration) or equal to (if the bodies touch one another exactly at one point) zero. Let us describe the Figure 7 in more details. Initially the top smaller ball is out of contact, and corresponding indicator is positive, dashed curve. But other indicator is not equal to zero. Instead it is negative, see the vertically scaled subfigure at the upper edge of Figure 7. Here at the very left side we see that initially indicator set to be of the zero value. Then the penetration develops and the whole top sinks into the floor by very small depth until the vertical quasi-equilibrium is reached. After that we can observe the vertical micro-oscillations develop into the modulated pulse decreasing afterwards. One can match the problem parameters such that the pulse amplitude will grow and the top can start to bounce over the floor thus distorting all the following dynamical predictions of its revolution. In the upper subfigure we can observe also the change of the balls at contact, before the instant of time $=2$ seconds. Then for the case of the smaller ball contacting the floor we observe the larger depth of penetration. Indeed, in this case we have a smaller area of the contact spot.

The bottom subfigure reflects the revolution process inself. Here the whole graph zoomed in vicinity of the time instant of 1.825 seconds, and we see that the revolution process is implemented by two attempts: two times the solid humps alternate the dashed ones. Thus first time the Tippe-Top "head" touches the floor then it once more is forced to loose a contact temporarily, and only then the head-floor contact becomes permanent. The right subfigure illustrates the depth of penetration for the larger and smaller balls by the vertical rescaling over the same interval as for the bottom subfigure.

In addition, yet another verification procedure has been performed, this time using the results of the paper [10] (one can access the paper [10] using the ScienceDirect on-line library [22]). Namely, exact formulae for the friction force and for the drilling friction torque, case (a), were applied to the top dynamics computer model implemented on Modelica language in frame of the unrestricted, in sense mentioned above, 


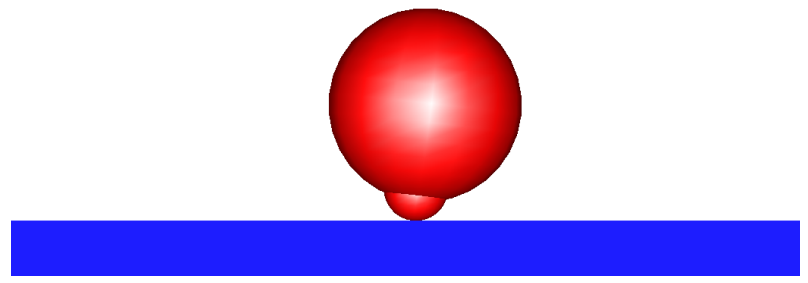

Figure 9: The Tippe-Top 3D-animation

contact model. In the same dynamical frame the simplified Contensou model, case (b), as well as a linearfractional Pade approximation for the friction force and torque, case (c), were also implemented. The results of the inclination angle evolution are shown in Figure 8, where the cases (a), (b), and (c) correspond to the solid, dashed, and dotted curves respectively. One can see easily the revolution scenarios are mutually closest in cases (a) and (b). The 3D-animation shot is shown in Figure 9.

Note in addition, one can easily obtain a behavior typical to the Tippe-Top, revolution to "head", in frame of the "regularized" Amontons-Coulomb friction. One has to understand regularization in a sense proposed in the works $[8,11]$ and used in [4] in case of the point contact. We only have to "bend" graph for the friction force dependence on the relative slip velocity in vicinity of zero replacing its discontinuity by the linear function. The more flat slope of the graph the sooner one can find out the Tippe-Top revolution effect. As the simplified Contensou model shows that just this slope appears in the corresponding graph for the friction force dependence on the velocity, this time in frame of the exact Contensou-Erismann model.

The dynamical model of the ball bearing was considered in a way similar to the paper [3] while the verification second stage. This time the contact area is essentially elliptic one. The main goal for the numeric simulations was to compare two approaches: (a) the standard Hertz model for the normal force plus the Contensou simplified model for the friction forces; (b) the simplified model of V. G. Vil'ke for the normal elastic force plus the Contensou simplified model for the friction forces. As it was observed in [3] for the case of the regularized Coulomb friction force here dynamical models of the cases (a) and (b) differ one from another in a slightest degree too. Simultaneously, the model (b) is faster than (a) by $20 \%$ meaning the CPU time needed. To illustrate this in Figure 10 we compare the cases (a) and (b) for one component of the tangent friction force at a contact between one of the bearing ball and its inner raceway. The solid curve corresponds to the case (a) while the dashed one represents the case (b). A values of the contact spot eccentricity squared appeared to be constant equal to 0.687 in the case (a) and 0.643 in the case (b).

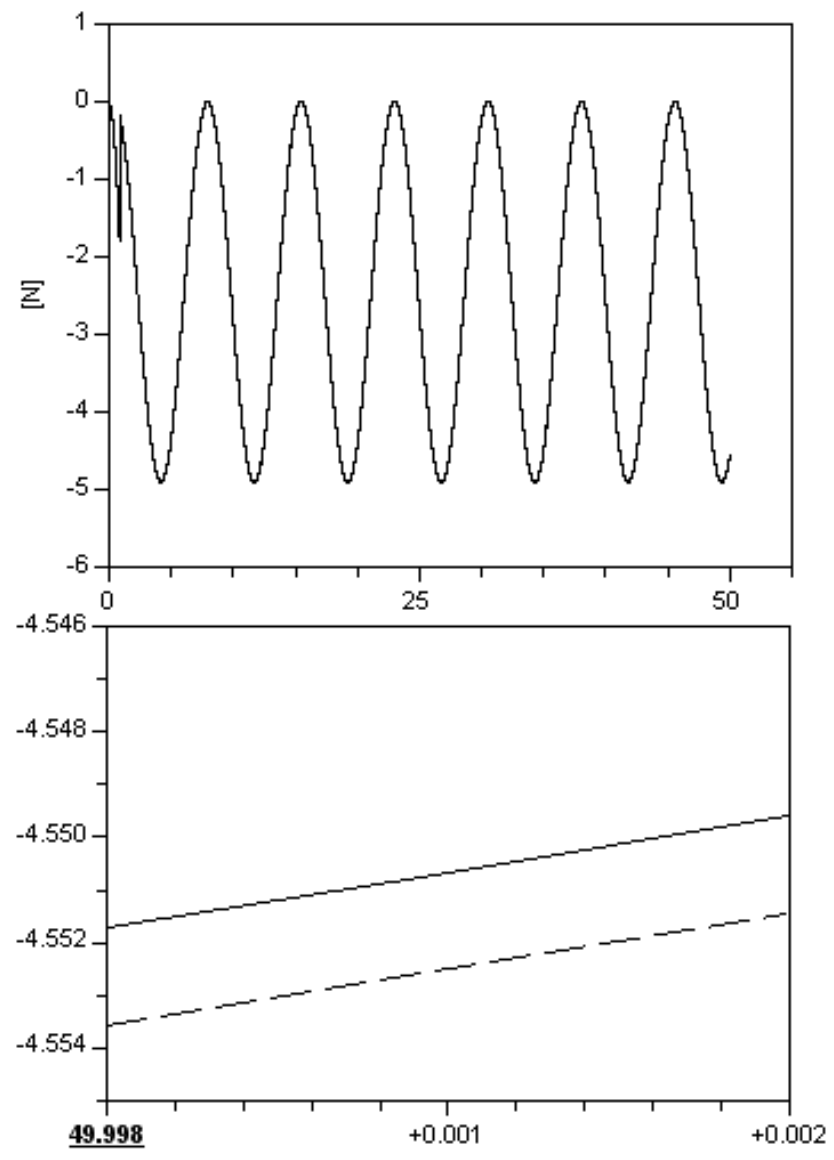

Figure 10: One of the friction force component evolution. A final stage of the simulation zoomed in.

\section{Conclusions}

Summarizing the results described above we can remark the following.

- The Tippe-Top "on head" revolution effect is caused completely by the dry friction force "regularization" in vicinity of zero value for the velocity of relative slip. Such a regularization takes place exactly in the Contensou-Erismann model. Numeric experiments showed if the slope of friction force graph in vicinity of the zero velocity in the regularized Coulomb model is steep enough then the Tippe-Top effect either isn't observed at all or arising during short time after a long evolution then vanishes quickly. And only noticeable decreasing of the slope mentioned immediately 
causes the top revolution on the "head" with the subsequent long precession in this position.

The Contensou-Erismann model creates a property just as one described above. Note the drilling friction torque role is reduced to a dissipative effect with subsequent gradual "fall" of the top approaching it to the static stable configuration.

- Since complete elliptic integrals used in the Contensou simplified model are already found in frame of the Hertz algorithm while computing the normal force then from the computational viewpoint application of this model is practically "cost free". If, in addition, we will take into account an effect of the regularization provided by the Contensou-Erismann model then we arrive at a unexpected from the first sight result: a numeric simulation of the Hertz model for the normal force and the Contensou-Erismann for the tangent force and the drilling friction torque turned out to be faster than the combination of the Hertz model and the "simple" Amontons-Coulomb dry friction. It is evident such a deceleration in latter case surely concerns the large stiffness of the problem while the almost rolling mode.

- Though for isotropic case, one of the circular contact area, the tangent forces average values for the Amontons-Coulomb and ContensouErismann models differ not so much, however in anisotropic case the first model becomes inadequate while the second one continues to serve correctly the contacting process simulation. Such a property has an importance for instance in case of the ball bearing simulation with the contact areas of essentially elliptic form.

Regarding the directions of a future work one can enumerate possible development and testing for different kinds of the contact properties combinations: normal-elastic-force / normal-viscous-force / tangentforce+drilling-friction-torque to match various engineering applications. It would be for instance different types of lubrication, or any new types of the normal elastic volumetric models etc.

\section{Acknowledgement}

The paper was prepared with partial support of Russian Foundation for Basic Research, projects 08-0100600-a, 08-01-00718-a, 08-08-00553-a.

\section{References}

[1] Contensou, P., Couplage entre frottement de glissement et frottement de pivotement dans la théorie de la toupie. In: Kreiselprobleme Gyrodynamics: IUTAM Symposium Celerina, 1962, Berlin: Springer, 1963, pp. 201-216.

[2] Erismann, Th., Theorie und Anwendungen des echten Kugelgetriebes. Z. angew. Math. Phys., 1954, Vol. 5, No. 5, pp. 355-388.

[3] Kosenko I. I., Alexandrov E. B., Implementation of the Hertz Contact Model and Its Volumetric Modification on Modelica. In: Bachmann, B. (Ed.) Proceedings of the 6th International Modelica Conference, Bielefeld, Germany, March 3-4, 2008, Bielefeld: The Modelica Association, and University of Applied Sciences Bielefeld, 2008, pp. 203-212.

[4] Kossenko, I. I., Implementation of Unilateral Multibody Dynamics on Modelica. In: Schmitz, G. (Ed.) Proceedings of the 4th International Modelica Conference, Hamburg-Harburg, Germany, March 7-8, 2005, Hamburg-Harburg: The Modelica Association, and The Department of Thermodynamics, Hamburg University of Technology, 2005, pp. 13-23.

[5] Hertz, H., Über die Berührung fester elastischer Körper. J. reine und angewandte Mathematik, 1882, B. 92, S. $156-171$.

[6] Landau, L. D. and Lifshitz, E. M., Theory of Elasticity. 3rd Edition. Landau and Lifshitz Course of Theoretical Physics. Volume 7. Oxford - Boston - Johannesburg - Melbourne - New Delhi - Singapore: Reed Educational and Professional Publishing Ltd., 1999.

[7] Leine, R. I. and Nijmeijer, H., Dynamics and Bifurcations of Non-Smooth Mechanical Systems. Berlin - Heidelberg - New York: Springer Verlag, 2004.

[8] Novozhilov, I. V., Conditions of Stagnation in Systems with the Coulomb Friction. Mechanics of Solids, 1973, Vol. 8, No. 1, pp. 8-14.

[9] Novozhilov, I. V., Fractional Analysis: Methods of Motion Decomposition, Boston: Birkhauser, 1997. 
[10] Zhuravlev, V. F., The Model of Dry Friction in the Problem of the Rolling of Rigid Bodies. J. Appl. Math. Mech., 1998, Vol. 62, No. 5, pp. $705-710$.

[11] Rooney, G. T. and Deravi, P., Coulomb Friction in Mechanism Sliding Joints. Mechanism and Machine Theory, 1982, Vol. 17, Iss. 3, pp. 207211.

[12] Kireenkov, A. A., Three-Dimensional Model of Combined Dry Friction and Its Application in Non-Holonomic Mechanics. In: van Campen, D. H., Lazurko, M. D. van den Oever, W. P. J. M., (Eds.) Proceedings of ENOC2005, Fifth EUROMECH Nonlinear Dynamics Conference, Eindhoven, August 7-12, 2005, Eindhoven, The Netherlands: Eindhoven University of Technology, 2005.

[13] Aleksandrov, E. B., Vil'ke, V. G. Kosenko, I. I., Hertzian Contact Problem: Numerical Reduction and Volumetric Modification. Computational Mathematics and Mathematical Physics, 2008, Vol. 48, No. 12, pp. 2226-2240.

[14] Whittaker, E. T., Watson, G. N., A Course of Modern Analysis, Cambridge - New York - Melbourne - Madrid - Cape Town: Cambridge University Press, 2002.

[15] Janke, E., Emde, F., Lösch, F., Tafeln Höherer Funktionen, Stuttgart: B. G. Teubner Verlagsgesellschaft, 1960.

[16] Milne-Thomson, L. M., Elliptic Integrals, Abramowitz, M., Stegun, I. A., (Eds) Handbook of Mathematical Functions: with Formulas, Graphs, and Mathematical Tables, New York: Dover Publications Inc., 1972.

[17] Bateman, H., Erdélyi, A., Higher Transcendental Functions. Volume 3. New-York - Toronto - London: Mc Graw-Hill Book Company, Inc., 1955.

[18] Kosenko, I. I., Loginova, M. S., Obraztsov, Ya. P. and Stavrovskaya, M. S., Multibody Systems Dynamics: Modelica Implementation and Bond Graph Representation. In: Kral, Ch. and Haumer, A., (Eds.) Proceedings of the 5th International Modelica Conference, Vienna, Austria, September 4-5, 2006, Vienna: The Modelica Association, and arsenal research, 2006, pp. 213223.
[19] Leine, R. I. and Glocker, Ch., A Set-Valued Force Law for Spatial Coulomb-Contensou Friction. Europian Journal of Mechanics A/Solids, 2003, Vol. 22, No. 2, pp. 193-216.

[20] Friedl, C., Der Stehaufkreisel. Master's thesis. Augsburg: Institut für Physik, Universität Augsburg, 1997.

[21] http://www.zfm.ethz.ch/ leine/ publications.htm

[22] http://www.sciencedirect.com/ science/journal/00218928 